\title{
Der Stellenwert der Ethik in unserer Gesellschaft
}

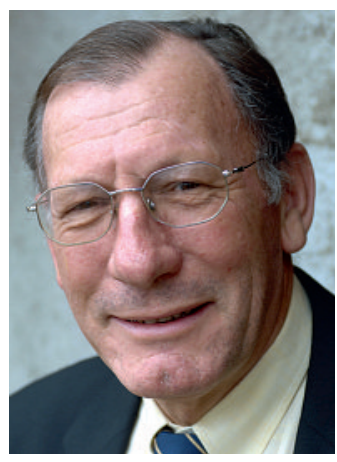

Jean Martin
Vor kurzem schrieb der Journalist Beat Kappeler: «Die Nationale Ethikkommission hat keinen Präsidenten mehr und Pascal Couchepin zögert mit der Ernennung eines Nachfolgers. Wie Couchepin hege auch ich meine Zweifel am Nutzen und an der Legitimität solcher Kommissionen [...]. Es gibt keine Instanz in der Gesellschaft, die das Wissen über die oberste und unanfechtbare Ethik besitzt und ihr daher eine solche auferlegen könnte.» [1]

Es ist sehr bedauerlich, dass dieser in den Medien stark präsente Beobachter so schlecht informiert ist und eine Meinungsäusserung abdrucken lässt, die das Mandat einer Nationalen Ethikkommission (NEK) nicht einmal ansatzweise erfasst. Es wäre wünschenswert auch im Sinne deontologischer Grundsätze, wie z.B. die Gesundheitsberufe sie haben - dass sich ein Journalist ein paar Minuten Zeit nimmt, um sich mit dem Thema zu befassen, über das er zu schreiben gedenkt (zum Beispiel auf www.nek-cne.ch), bevor er der Öffentlichkeit professorale Lektionen zuteil werden lässt. Den Gesetzestexten zur Einsetzung der Ethikkommission zufolge verfolgt die Kommission «die Entwicklung der Wissenschaften über die Gesundheit und Krankheit des Menschen und ihrer Anwendungen. Sie nimmt zu den damit verbundenen gesellschaftlichen, naturwissenschaftlichen und rechtlichen Fragen aus ethischer Sicht beratend Stellung». Eine Mission ohne jeden Sinn? Kann man einfach negieren, dass die Fortschritte in der Biomedizin grundlegende ethische Werte in Frage stellen?

Die Mitglieder der Kommission werden vom Bundesrat ad personam bestellt und arbeiten vollkommen unabhängig. Dies ist eine der grundlegenden Bedingungen aller Kommissionen dieser Art weltweit. Nebenbei wird man feststellen, dass die Schweiz im Falle einer Abschaffung der Ethikkommission wieder einmal ihren Status als «Sonderfall» unter Beweis stellen würde, denn alle mit uns vergleichbaren Länder haben eine solche Instanz auf nationaler Ebene und unterstützen diese deutlich stärker. Aber vielleicht sind sie ja alle im Irrtum?

Diese Kommissionen geben keine verbindlichen Bescheide $\mathrm{ab}$, sondern sprechen als Konsultativorgan Stellungnahmen und Empfehlungen aus, die sich an Behörden und an die gesamte Gesellschaft richten. Ferner können diese Äusserungen an bestimmte Berufsgruppen, Bildungseinrichtungen oder auch Wirtschaftskreise gerichtet sein [2].

Grundsätzlich kann man sich die Frage stellen, wie - gerade in der heutigen Zeit - jemand zum Schluss kommen kann, dass hochrangige beratende Organe/ Kommissionen, deren Aufgabe die Erörterung die gesamte Gemeinschaft betreffender ethischer Fragen ist (und die neben so vielen anderen, nach Meinung Kap- pelers ausschliesslich den ernsthaften Gebieten, wie dem Business, gewidmeten Kommissionen bestehen), kostspielig und entbehrlich sind. Sicherlich ist angesichts gewisser Modeerscheinungen eine kritische Distanz angebracht, denn es ist richtig, dass die Aspekte der «Ethik» in den letzten Jahren auffallend häufig Erwähnung finden. Doch oft hat dies durchaus Berechtigung. So werden z.B. viele Polizeikorps von Ethikkommissionen oder -beratern unterstützt, und die Leitungsebenen bestätigen, dass diese wertvolle Dienste leisten.

Und was ist vom folgenden Satz Kappelers zu halten: «Die in diesen Tagen veröffentlichten Jahresrechnungen der Banken verstärken den Eindruck, dass man hier nicht gleich ethische Grundsatzfragen stellen muss. Den Verantwortlichen sind schwerwiegende Kunstfehler anzulasten - das ist alles.»? Der Leser begrüsst besonders das französische «tout simplement» - das ist alles. Die Welt steckt in einer durch den Mangel der Grössten der Finanzbranche an einer angemessenen berufsständischen Ethik verursachten tiefen Krise, und es gibt anscheinend keinen Bedarf an unabhängigen Ethikorganen. Die Wirtschaft würde ihre Fehler aus eigener Kraft beheben ... Möglich, aber erst nachdem alles Porzellan zerschlagen wurde und in keinem Fall präventiv. Ist es in Frage zu stellen (oder sogar dumm?), wenn man den Wunsch äussert, solchen Krisen sollte so weit irgend möglich vorgebeugt werden? Dies gilt für die Wirtschaft, aber ganz klar auch für andere Gebiete, wie die Biomedizin, auf die die NEK ihr Hauptaugenmerk richtet.

Ich bin nicht der Einzige, der es für notwendig erachtet, neben der unsichtbar lenkenden Hand des Marktes und der Gewinnsucht der Industriemagnaten von der Regierung eingesetzte ethische Instanzen mit eindeutigem Auftrag zu haben. Eine solche Instanz bildet ein (wohltuendes, wenngleich bescheidenes) Gegengewicht zu dem auf zu simplen, auf das eigene Interesse zentrierten Kriterien begründeten Denken all jener, die sich als Unternehmer bezeichnen. Und zwar zu einem Zeitpunkt, an dem eine Wirtschaftsredakteurin titelt: «New York wieder im Fieber: Es muss weitergehen, aber wie bisher» [3].

Diese Instanzen beleuchten die heutigen und künftigen Herausforderungen aus einem Blickwinkel, der weder opportunistisch noch durch Einzelinteressen (finanzieller Art) motiviert ist und nicht durch die Interessenkonflikte infiziert. Sie sind nicht Teil des «Filzes» (Beziehungsgeflechte und gegenseitiges «Erkenntlichzeigen» in Politik und Wirtschaft). Brauchen wir sie wirklich nicht? Allein die Frage zu stellen, ist bereits die Antwort.

Dr. med. Jean Martin, Mitglied der Redaktion und der Nationalen Ethikkommission 